\title{
Memória espacial e/ou olfativa em flebotomíneos em área endêmica de leishmaniose tegumentar americana, sul do Brasil
}

\author{
Spatial and/or olfactory memory in sandflies in an endemic area for American \\ cutaneous leishmaniasis, southern Brazil
}

\author{
Janaína Sales de Freitas, Kárin Rosi Reinhold-Castro, Cláudio Casanova², \\ Joseane Padilha da Silva ${ }^{3}$, Isolde Previdelli ${ }^{3}$ e Ueslei Teodoro ${ }^{1}$
}

\begin{abstract}
RESUMO
Relata-se os resultados de uma investigação sobre a possibilidade da existência de memória em flebotomíneos em área endêmica de leishmaniose tegumentar americana, no Estado do Paraná. Capturaram-se flebotomíneos no Recanto Marista, Doutor Camargo, Estado do Paraná, Brasil, com armadilhas de Falcão, de 15/11/2007 a 26/11/2007, em dois galinheiros (G1 e G2). Foram capturados 2.080 flebotomíneos (1.000 em G1 e 1.080 em G2), que foram marcados e soltos. Nyssomyia neivai foi a espécie mais (90,5\%) freqüente. Do total solto, recapturaram-se 168 (8\%) flebotomíneos e a recaptura no G2 foi significativa. Os resultados evidenciam que é possível a existência de memória espacial, olfativa e/ou a fidelidade ao hospedeiro e que isto orientem os flebotomíneos no reconhecimento dos locais onde há disponibilidade de fontes de sangue.
\end{abstract}

Palavras-chaves: Leishmaniose tegumentar americana. Memória em flebotomíneos. Epidemiologia.

\section{ABSTRACT}

The results from an investigation on the possibility that sandflies in an endemic area for American cutaneous leishmaniasis, in the state of Paraná, may have memory are reported. Sandflies were caught in Recanto Marista, Doutor Camargo, State of Paraná, Brazil, using Falcão traps in two chicken sheds (G1 and G2), between November 15 and 26, 2007. A total of 2,080 sandflies were caught (1,000 in G1 and 1,080 in G2) and these were marked and released. Nyssomyia neivai was the most (90.5\%) frequent species. Out of the total released, 168 sandflies (8\%) were recaptured and the recapture rate in G2 was significant. The results show that it is possible that spatial or olfactory memory and/or host loyalty exists, and that this will guide the sandflies in recognizing the places where sources of blood are available.

Key-words: American cutaneous leishmaniasis. Memory in sandflies. Epidemiology.

A leishmaniose é uma das doenças infecto-parasitárias de maior incidência no mundo, com presença em 88 países, onde afeta aproximadamente 14 milhões de pessoas e 350 milhões vivem em área de risco ${ }^{31}$.

A epidemiologia dessa parasitose, causada por protozoários do gênero Leishmania, envolve obrigatoriamente mamíferos (reservatórios) e flebotomíneos (vetores), em ambientes e climas $\operatorname{diversos}^{10}$.

No Brasil, a leishmaniose tegumentar americana (LTA) tem sido notificada em todos os estados ${ }^{19}$. No Estado do Paraná, há registros de casos na maioria dos municípios ${ }^{14}$. Dos 13.486 casos

\footnotetext{
1. Programa de Pós-Graduação em Análises Clínicas, Universidade Estadual de Maringá. Maringá, PR. 2. Superintendência de Controle de Endemias (SUCEN). Mogi Guaçu, SP. 3. Departamento de Estatística da Universidade Estadual de Maringá. Maringá, PR. Apoio financeiro: Fundação Araucária (convênio 225/2005), CNPq (Processo 4105502006-0) e Coordenação de Aperfeiçoamento de Pessoal de Nível Superior (CAPES). Endereço para correspondência: Dr. Ueslei Teodoro. Depṭ de Análises Clínicas/UEM. Av. Colombo 5790, 87020-900 Maringá, PR.

Tel: 5544 3261-3783; Fax: 5544 3261-4860

e-mail: uteodoro@uem.br

Recebido para publicação em 21/08/2008

Aceito em 20/03/2009
}

registrados na região Sul do país, no período de 1980 a 2005, 98,7\% ocorreram no Paraná ${ }^{19}$.

Mediadores químicos são utilizados por vertebrados e invertebrados na comunicação entre os indivíduos. Os semioquímicos abrangem substâncias intra-específicas (feromônios) e interespecíficas (infoquímicos), destacando-se dentre estes os cairomônios ${ }^{30}$. De acordo com Kelly e Dye ${ }^{13}$, os feromônios dos machos e os cairomônios (odores) dos hospedeiros vertebrados funcionam como atrativos para a realização do repasto sanguíneo pelas fêmeas. 0 estudo sobre flebotomíneos desenvolvido no Paraná por Pinto e cols ${ }^{21}$, o único que avalia a atratividade de flebotomíneos pelo dióxido de carbono e odor humano, não menciona a possibilidade da existência de memória em flebotomíneos. Para melhor entender o comportamento de retorno ao local de repasto e reprodução, experimentos de marcação-soltura-recaptura têm sido utilizados para evidenciar a memória de alguns insetos, o que vai além da atração pelos odores.

Evidências de memória em insetos hematófagos já foram demonstradas por Charlwood e cols ${ }^{5}$, em Papua (Nova Guiné), verificando que o mosquito Anopheles farauti fazia longos voos para a oviposição, mas retornava ao local onde já havia se 
alimentado de sangue. McCall e Eaton ${ }^{15}$ sugeriram a memória olfativa para Culex quinquefasciatus e Kaur e cols ${ }^{11}$, para Aedes aegypti. 0 comportamento de agregação e retorno aos locais de hematofagia também foi registrado em Lutzomyia longipalpis, na Ilha de Marajó, no Estado do Pará12 e em Refrigério no Estado do Pernambuco, Campbell-Lendrum e cols ${ }^{3}$ observaram um retorno significativo de Lutzomyia whitmani aos abrigos de animais domésticos.

Neste estudo, procurou-se investigar a existência de memória em flebotomíneos em área endêmica para LTA, no Estado do Paraná, visando obter informações que possam contribuir para evitar a invasão do domicílio por esses insetos.

\section{MATERIAL E MÉTODOS}

A pesquisa foi realizada no Recanto Marista, às margens do Rio Ivaí, numa área de lazer, com mata remanescente de floresta estacional, situada no município de Doutor Camargo, que está inserido na Mesorregião Norte Central do Paraná, a 52 ${ }^{\circ} 13$ ' Longitude Oeste e $23^{\circ} 33^{\prime}$ Latitude Sul.

No período de captura e recapturas de flebotomíneos, a temperatura e a umidade relativa do ar foram verificadas às 20 horas, à 1 hora e às 6 horas. A temperatura média dos dias de recapturas foi de $24,3^{\circ} \mathrm{C}$, com a mínima de $19,7^{\circ} \mathrm{C}$ e máxima de $31,7^{\circ} \mathrm{C}$. A umidade relativa média do ar foi de $69 \%$, com a mínima de $40 \%$ e máxima de $99 \%$.

A captura de flebotomíneos foi realizada em 15/11/2007 e as recapturas de flebotomíneos no período de 16/11/2007 a 26/11/2007, com armadilhas de Falcão, em dois galinheiros (G1 e G2), cada um com duas galinhas e um galo, a 90m de distância um do outro (Figura 1). Esta condição foi mantida, para que os flebotomíneos tivessem a mesma chance de retornar a qualquer um dos galinheiros. Além disso, entre os dois galinheiros não havia barreiras (edificações) que poderiam interferir nas recapturas. Outro motivo da escolha dos pontos de coletas foram os resultados de um estudo prévio de dispersão de flebotomíneos realizado no Recanto Marista, com o uso da metodologia de marcação-soltura-recaptura, mostrando que 251 (70,3\%) flebotomíneos foram recapturados no $\mathrm{G1}^{7}$.

$\mathrm{Na}$ primeira noite do experimento, após a captura, as armadilhas de cada um dos galinheiros foram abertas dentro de gaiolas de onde os flebotomíneos foram sugados com o aspirador de Castro, contados e imediatamente transferidos para caixas de isopor forradas com gesso umedecido, semelhante à usada por Casanova e cols ${ }^{4}$, para conservá-los vivos. Para cada galinheiro foi utilizada uma gaiola e uma caixa de isopor. Em seguida, o pó fluorescente (Bioquip Inc) foi aspergido sobre os flebotomíneos, marcando-os de vermelho (G1) e amarelo (G2).

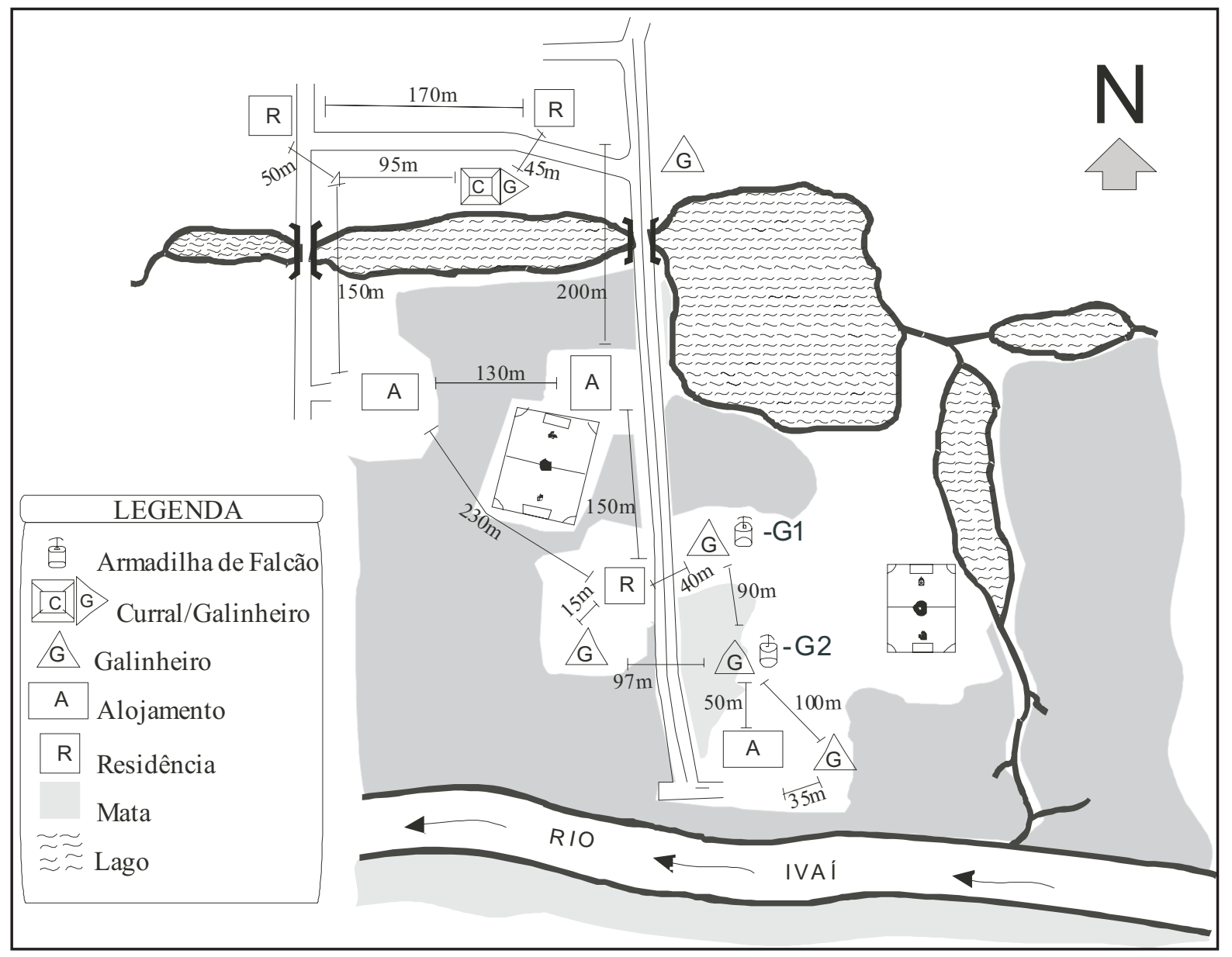

FIGURA 1

Localização dos galinheiros (G1 e G2) onde foram feitas as capturas e recapturas de flebotomíneos no Recanto Marista, Doutor Camargo, Estado do Paraná, no período de 15 a 26 de novembro de 2007. 
Os flebotomíneos foram soltos entre os dois galinheiros (G1 e G2), a 45m de distância de cada um. As recapturas de flebotomíneos foram feitas nos dois galinheiros, nas 11 noites subseqüentes à captura, com uma armadilha de Falcão instalada em cada galinheiro, das 20 horas às 6 horas. As armadilhas foram substituídas à 1 hora, pois a carga das baterias durava em torno de 6 horas. Depois de recapturados, os flebotomíneos foram mortos com clorofórmio e examinados em estereomicroscópio sob black light blue (luz negra) para isolar os exemplares marcados, que foram posteriormente identificados.

A nomenclatura das espécies de flebotomíneos segue Galati ${ }^{8}$.

Para a análise estatística, utilizou-se a metodologia dos Modelos Lineares Generalizados (GLM). Adotou-se para a variável resposta, a distribuição binomial, uma vez que esta distribuição descreve melhor o comportamento dos dados em estudo e uma função de ligação $\log ^{620}$. A análise dos dados foi executada na biblioteca $G L M$ da linguagem de programação estatística "R", versão 3.5. Em todas as análises considerou-se um nível de significância de 5\%.

\section{RESULTADOS}

Na Tabela 1, observa-se que foram marcados 2.080 flebotomíneos (1.000 de G1 e 1.080 de G2). Foram recapturados 168 (8\%) flebotomíneos, 97 (57,7\%) fêmeas e 71 (42,3\%) machos, das espécies Nyssomyia neivai, Nyssomyia whitmani, Migonemyia migonei, Pintomyia pessoai e Psathyromyia shannoni, representados especialmente pela primeira espécie (90,5\%).
Recapturou-se 52 exemplares no G1 e 116 no G2. No G1, foram recapturados 29 flebotomíneos, 16 fêmeas e 13 machos, que foram capturados originalmente neste galinheiro. No G1, foram também recapturados 23 flebotomíneos, 12 fêmeas e 11 machos, que haviam sido capturados no G2. No G2, recapturaram-se 70 flebotomíneos, 38 fêmeas e 32 machos, que foram capturados originalmente neste galinheiro. Ainda no G2, recapturaram-se 46 flebotomíneos, 31 fêmeas e 15 machos, que haviam sido capturados em G1.

Os resultados da análise estatística mostram que não houve diferença significativa entre os flebotomíneos recapturados em G1 (29) e os recapturados em G1 (23), mas que haviam sido capturados em G2. No entanto, o número de flebotomíneos recapturados em G2 (70) difere do número de flebotomíneos recapturados em G2 (46), mas que haviam sido capturados em G1 $(\mathrm{p}<0,05)$.

A chance dos flebotomíneos serem recapturados em seu galinheiro de origem é estatisticamente maior em G2 ( $<<0,001)$ e a recaptura destes em $\mathrm{G} 2$ é aproximadamente de 2,45 vezes maior do que em G1 $(\mathrm{p}<0,001)$. A chance de um flebotomíneo que foi recapturado em G2 ter vindo do próprio G2 é de 1,52 maior que a chance dele ter vindo de $\mathrm{G} 1(\mathrm{p}<0.002)$.

0 número de flebotomíneos recapturados em G2 (70) foi significativamente maior do que o recapturado em G1 (29) $(\mathrm{p}<0,001)$. Houve diferença significativa apenas entre os flebotomíneos fêmeas e machos recapturados em G2 $(\mathrm{p}<0,05)$.

A maioria dos flebotomíneos foi recapturada na primeira noite de recaptura (47 exemplares) e na segunda (65 exemplares).

TABELA 1

Flebotomíneos recapturados no Recanto Marista, Doutor Camargo, Estado do Paraná, Brasil, no período de 16 a 26 de novembro de 2007.

\begin{tabular}{|c|c|c|c|c|c|c|c|c|c|c|c|}
\hline \multirow{3}{*}{$\begin{array}{l}\text { Local de captura } \\
\text { Local de recaptura } \\
\text { Noites de recaptura }\end{array}$} & \multirow{2}{*}{\multicolumn{2}{|c|}{$\begin{array}{l}\text { G1* } \\
\text { G1 }\end{array}$}} & \multirow{2}{*}{\multicolumn{2}{|c|}{$\begin{array}{l}\mathrm{G} 2 \\
\mathrm{G} 1 \\
\end{array}$}} & \multirow{2}{*}{\multicolumn{2}{|c|}{$\begin{array}{l}\mathrm{G} 2^{* *} \\
\mathrm{G} 2\end{array}$}} & \multicolumn{2}{|c|}{ G1 } & \multirow{2}{*}{\multicolumn{3}{|c|}{ Recaptura/dia }} \\
\hline & & & & & & & & & & & \\
\hline & $\mathrm{F}$ & M & $\mathrm{F}$ & M & $\mathrm{F}$ & M & $\mathrm{F}$ & M & $\mathrm{F}$ & M & Total \\
\hline 1 & 8 & 4 & 12 & 11 & 3 & 2 & 7 & - & 30 & 17 & 47 \\
\hline 2 & - & - & - & - & 32 & 23 & 7 & 3 & 39 & 26 & 65 \\
\hline 3 & - & - & - & - & 1 & 4 & 2 & 2 & 3 & 6 & 9 \\
\hline 4 & 1 & 3 & - & - & - & 1 & 1 & - & 2 & 4 & 6 \\
\hline 5 & 1 & 2 & - & - & 1 & - & 12 & 6 & 14 & 8 & 22 \\
\hline 6 & - & - & - & - & - & 1 & 2 & 2 & 2 & 3 & 5 \\
\hline 7 & 6 & 3 & - & - & 1 & - & - & - & 7 & 3 & 10 \\
\hline 8 & - & - & - & - & - & - & - & 1 & - & 1 & 1 \\
\hline 9 & - & - & - & - & - & 1 & - & - & - & 1 & 1 \\
\hline 10 & - & 1 & - & - & - & - & - & 1 & - & 2 & 2 \\
\hline 11 & - & - & - & - & - & - & - & - & - & - & - \\
\hline \multirow[t]{2}{*}{ Total } & 16 & 13 & 12 & 11 & 38 & 32 & 31 & 15 & 97 & 71 & 168 \\
\hline & \multicolumn{4}{|c|}{52} & \multicolumn{4}{|c|}{116} & \multicolumn{2}{|c|}{168} & \\
\hline
\end{tabular}

${ }^{*}$ G1: neste galinheiro foram capturados e marcados 1.000 flebotomíneos, ${ }^{* * *} \mathrm{G} 2$ : neste galinheiro foram capturados e marcados 1.080 flebotomíneos. F: fêmea, M: macho. 


\section{DISCUSSÃo}

Os flebotomíneos recapturados representaram $8 \%$ do total capturado, resultado comparável com os dados registrados em outros estudos ${ }^{1325}$.

A recaptura de maior proporção de flebotomíneos em G2 (69\%) pode ter sido devido a proximidade de possíveis locais diurnos de repouso dos flebotomíneos, uma vez que o $\mathrm{G} 2$ localizase próximo à mata, onde há maior disponibilidade de abrigos e a temperatura sofre menos alteração, comparada a localização do G1. Além disso, os flebotomíneos recapturados poderiam ter sido atraídos tanto para o G1, quanto para $0 \mathrm{G} 2$, pela presença de cairomônios produzidos pelos galináceos nos dois galinheiros, não se tratando, portanto, da fidelidade aos locais de repasto sanguíneo, mas da fidelidade ao hospedeiro e/ou graças à memória olfativa. A chance de recaptura no G2 foi significativamente maior do que no G1 e os flebotomíneos, recapturados no G2, mostraram ser capazes de retornar ao galinheiro onde previamente haviam se alimentado. Num estudo prévio de dispersão de flebotomíneos, realizado no Recanto Marista, com o uso da mesma metodologia de marcação-soltura-recaptura, a maior proporção de flebotomíneos recapturados foi no $\mathrm{G1}^{7}$, onde a maioria $(70,3 \%)$ deles tinha sido capturada para a marcação. Estes autores realizaram as capturas quando as fezes e os restos de alimentos, retirados de diversos galinheiros, foram utilizados como adubo numa horta muito próxima do G1 e isto pode ter possibilitado a formação de criadouros de flebotomíneos ao lado desse ecótopo (U Teodoro: dados não publicados). Este fato corrobora a possibilidade de memória olfativa e/ou espacial de flebotomíneos.

A identificação de alguns odores do hospedeiro pelos insetos pode ajudá-los também na escolha de um hospedeiro com menor capacidade de defesa, garantindo maior sucesso no repasto sanguíneo. Na África, constatou-se que as moscas tsé-tsé (Glossina spp) procuravam se alimentar em bovinos mais velhos e menos defensivos do que nos bezerros ${ }^{29}$, pois a experiência do repasto sanguíneo sem interrupção, pode tornar o hospedeiro mais atrativo ${ }^{12}$. 0 repasto sanguíneo ou as cópulas anteriores em determinados locais podem constituir experiências positivas, provavelmente, memorizadas por flebotomíneos, que em ocasiões subsequentes procurarão os mesmos locais, em função de experiências prévias bem sucedidas. McCall e cols ${ }^{17}$ observaram que Anopheles arabiensis retornava ao local onde havia feito o primeiro repasto sanguíneo, propondo que este mosquito seria capaz de uma memorização espacial. Machos de Drosophila mercatorum aprimoraram a habilidade de cortejar as fêmeas em função de experiências anteriores ${ }^{22}$. Charlwood e cols ${ }^{5}$, em estudo com Anopheles farauti, e Renshaw e cols ${ }^{25}$, com Aedes cantans, sugeriram que esses insetos são capazes de memorizar uma determinada área espacial que lhes facilite a circulação nos locais de repasto sanguíneo, repouso e reprodução. De acordo com McCall e Kelly ${ }^{16}$, os insetos são capazes de aprender ou memorizar informações dos locais de repasto sanguíneo, de postura de ovos e do abrigo do hospedeiro e que, talvez, sua capacidade de memorização seja genética, sendo a informação adquirida durante a vida, moldando o seu comportamento conforme as suas necessidades e os locais onde vivem.
Embora a memória dos insetos possa ser genética, McCall e Eaton ${ }^{15}$, ao condicionarem as larvas do mosquito Culex quinquefasciatus a uma solução repelente (água/skatole), observaram que as fêmeas preferiram ovipor na mesma solução. Porém, a geração F1 desse inseto não herdou esse comportamento dos pais, demonstrando que as preferências para oviposição foram adquiridas e não herdadas. Resultados semelhantes foram observados por Kaur e cols ${ }^{11}$ que reduziram a aversão de Aedes aegypti ao repelente Mozaway ${ }^{\mathrm{TM}}$ (citronela e nim), caracterizando este fato como aprendizagem. Entretanto, Alonso e cols² não constataram evidências de aprendizagem em Aedes aegypti, na tentativa de condicioná-lo a determinados estímulos (sangue, respiração humana, vibração e choque elétrico), odores particulares (citral, citronela e eugenol) e padrões visuais (barras pretas horizontais ou verticais). Campbell-Lendrum e cols ${ }^{3}$ sugeriram uma possível heterogeneidade de comportamento de flebotomíneos relativa à preferência por um ou outro hospedeiro.

Nas recapturas, a proporção de fêmeas de flebotomíneos foi estatísticamente maior do que a de machos no galinheiro G2. Fato já observado na mesma localidade onde este trabalho foi realizado $^{2327}$. Porém, énotável a recaptura de machos nos galinheiros, principalmente no G1, onde não houve diferença estatística entre os sexos. Apesar dos machos não realizarem o repasto sanguíneo, sua abundância perto do hospedeiro é sempre correlacionada com a abundância de fêmeas. Campbell-Lendrum e cols ${ }^{3}$ relatam que a maior proporção de machos de Nyssomyia whitmani, presentes em abrigos de animais domésticos, pode ser atribuída ao fato de que as fêmeas procuram o hospedeiro a cada ciclo gonotrófico, de 3 a 4 dias, para um novo repasto sanguíneo, enquanto os machos procuram o hospedeiro todas as noites a fim de se acasalarem com as fêmeas. Contudo, estes autores ${ }^{3}$ ressalvam que o procedimento experimental por eles adotado pode ter causado mortalidade elevada entre as fêmeas, refletindo a baixa recaptura. Kelly e Dye ${ }^{13}$ observaram que os machos de Lutzomyia longipalpis eram atraídos pelo odor do hospedeiro e chegavam aos galinheiros antes das fêmeas, pois os feromônios liberados por eles devem ter contribuído para a agregação de outros machos, além de atrair as fêmeas. Os mesmos autores ${ }^{12}$ sugeriram que possivelmente esses insetos sejam capazes de lembrar dos locais de agregação. Embora se saiba que flebotomíneos de ambos os sexos sejam atraídos pela luz ${ }^{26}$, pode-se inferir com base nas observações de Gibson e Torr ${ }^{9}$ que este estímulo tem importância secundária para a localização do hospedeiro, devido à baixa percepção visual dos dípteros hematófagos.

Os resultados mostram que $66,7 \%$ dos flebotomíneos foram recapturados na primeira e segunda noites de recaptura. 0 número de flebotomíneos recapturados foi maior na segunda do que na primeira noite, provavelmente porque nesta ventava fortemente. Reisen e cols ${ }^{24}$ constataram que o vento foi um fator negativo na dispersão de Culex tarsalis. 0 aumento substancial do número de fêmeas recapturadas no quinto dia após a soltura pode estar relacionado com o retorno das mesmas, à procura de um novo repasto sanguíneo, após realizarem a oviposição. Casanova e cols ${ }^{4}$ estimaram para Nyssomyia neivai um ciclo gonotrófico de 4 dias, em condições naturais, no Estado de São Paulo. Éimportante ressaltar que o experimento em campo não permitiu mensurar 
a idade dos flebotomíneos marcados, contudo, é provável que a mortalidade natural desses insetos possa ter ocorrido ao longo dos dias de recapturas, refletindo no número de insetos recapturados. 0 pó fluorescente e a técnica de marcação, aqui adotados, que poderiam ser causas de mortalidade, não têm influência negativa sobre a taxa de sobrevivência dos flebotomíneos ${ }^{4}$.

A espécie Nyssomyia neivai tem sido registrada com frequiência, elevada neste Recanto ${ }^{232627}$, e em outras localidades no Estado do Paraná ${ }^{18}$. Por representar 90\% dos flebotomíneos coletados neste estudo, supõe-se que o comportamento observado seja especialmente referente a esta espécie.

O conhecimento dos parâmetros biológicos que conduzemà escolha do hospedeiro pode ser altamente pertinente para o planejamento do controle de doenças que envolvem insetos ${ }^{16}$. A heterogeneidade da população de flebotomíneos, a memória ou aprendizagem e fidelidade ao hospedeiro, necessitam ser melhor investigadas para que as ações de controle desses insetos sejam mais eficazes e efetivas. Na localidade deste estudo, pesquisas anteriores mostraram que o número deflebotomíneos é maior nos galinheiros do que em outros ecótopos ${ }^{2328}$, o que pode ser uma evidência a mais da existência de memória espacial, olfativa e/ou de fidelidade ao hospedeiro desses insetos.

Os resultados deste trabalho indicam que é possível a existência de memória espacial, olfativa e/ou a fidelidade ao hospedeiro e que isto possa orientar os flebotomíneos no reconhecimento dos locais onde há disponibilidade de fontes de sangue.

\section{AGRADECIMENTOS}

Os autores agradecem ao Colégio Marista pelo apoio logístico; ao João Eduardo Cavalcanti Brito e à Cristiane Machado de Oliveira Legriffon, pela ajuda nas coletas de flebotomíneos; ao João Balduíno Kühl, pelo auxílio na preparação do material de captura.

\section{REFERÊNCIAS}

1. Alexander B, Young DG. Dispersal of phebotomine sand flies (Diptera: Psychodidae) in a Colombian focus of Leishmania (Viannia) braziliensis. Memórias do Instituto Oswaldo Cruz 87: 397-403, 1992.

2. Alonso WJ, Wyatt TD, Kelly DW. Are vectors able to learn about their hosts? A case study with Aedes aegypti mosquitoes. Memórias do Instituto Oswaldo Cruz 98: 665-672, 2003

3. Campbell-Lendrum DH, Brandão-Filho SP, Ready PD, Davies CR. Host and/or loyalty of Lutzomyia whitmani (Diptera: Psychodidae) in Brasil. Medical and Veterinary Entomology 13: 209-211, 1999.

4. Casanova C, Costa AIP, Natal D. Dispersal pattern of the sand fly Lutzomyia neivai (Diptera: Psychodidae) in cutaneous leishmaniasis endemic rural area in Southeastern Brazil. Memórias do Instituto 0swaldo Cruz 100:719-724, 2005.

5. Charlwood JD, Graves PM, Marshall TFC. Evidence for a "memorized" host range in Anopheles farauti females in Papua New Guinea. Medical and Veterinary Entomology 2: 101-108, 1988.

6. Dobson A. An introduction to generalized linear models. Chapman \& Hall/CRC, Boca Raton, 1990.

7. Freitas JS, Reinhold-Castro KR, Casanova C, Brito JEC, Teodoro U. Estudo preliminar da dispersão de flebotomíneos no município de Doutor Camargo, Estado do Paraná, Brasil. In: Resumos da XXIII Reunião de Pesquisa Aplicada em doença de Chagas e Leishmanioses. Uberaba, MG, 2007.
8. Galati EAB. Morfologia e Taxonomia. In: Rangel EF, Lainson R (eds) Flebotomíneos do Brasil. Fundação Oswaldo Cruz, Rio de Janeiro, p. 23-51, 2003.

9. Gibson G, Torr SJ. Visual and olfactory responses haematophagous Diptera to host stimuli. Medical and Veterinary Entomology 13: 2-23, 1999.

10. Gontijo B, Carvalho MLR. Leishmaniose tegumentar Americana. Revista da Sociedade Brasileira de Medicina Tropical 36: 71-80, 2003.

11. Kaur JS, Lai YL, Giger AD. Learning and memory in the mosquito Aedes aegypti shown by conditioning against oviposition deterrence. Medical and Veterinary Entomology 17: 457-460, 2003.

12. Kelly DW. Why are some people bitten more than others? Trends in Parasitology 17: 578-581, 2001.

13. Kelly DW, Dye C. Pheromones, Kairomones and the aggregation dynamics of the sandfly Lutzomyia longipalpis. Animal Behaviour 53: 721-731, 1997.

14. Lima AP, Minelli L, Comunello E, Teodoro U. Distribuição da leishmaniose tegumentar por imagens de sensoriamento remoto orbital, no Estado do Paraná, Sul do Brasil. Anais Brasileiros Dermatologia 77: 681-692, 2002.

15. McCall PJ, Eaton G. Olfactory memory in the mosquito Culex quinquefasciatus. Medical and Veterinary Entomology 15: 197-203, 2001.

16. McCall PJ, Kelly DW. Learning and memory in disease vectors. Trends in Parasitology 18: 429-433, 2002

17. McCall PJ, Mosha FW, Njunwa KJ, Sherlock K. Evidence for memorized sitefidelity in Anopheles arabiensis. Transactions of the Royal Society of Tropical Medicine and Hygiene 95:587-590, 2001

18. Membrive NA, Rodrigues G, Membrive U, Monteiro WM, Neitzke HC, Lonardoni MVC, Silveira TGV, Teodoro U. Flebotomíneos de municípios do norte do estado do Paraná, sul do Brasil. Entomology Vectores 11: 673-680, 2004.

19. Ministério da Saúde. Leishmaniose Tegumentar Americana-Distribuição de casos confirmados de LTA de 1980 a 2005. http:/dtr2001.saude.gov.br/svs/epi/ situação_doencas/planilhas_doencas.htm (acessado em 12/12/2007).

20. Paula GA. Modelos de Regressão com apoio computacional. Editora da Universidade de São Paulo, São Paulo, 2004.

21. Pinto MC, Campbell-Lendrum DH, Lozovei AL, Teodoro U, Davies CR. Phlebotomine Sandfly responses to carbon dioxide and human odour in the field. Medical and Veterinary Entomology 15:132-139, 2001.

22. Polejack A, Tidon R. Learning of courtship components in Drosophila mercatorum (Paterson \& Wheller) (Diptera, Drosophilidae). Revista Brasileira de Entomologia 5: 82-86, 2007

23. Reinhold-Castro KR. Avaliação de medidas de controle de flebotomíneos no sul do Brasil. Dissertação de Mestrado.Universidade Estadual de Maringá. Maringá, Paraná. p.40, 2007.

24. Reisen WK, Lothrop HD, Lothrop B. Factors Influencing the outcome of markrelease-Recapture Studies with Culex tarsalis (Diptera: Culicidae) Journal of Medical Entomology 40: 820-829, 2003.

25. Renshaw M, Service MW, Birney MH. Host finding, feeding patterns and evidence for a memorized home range of the mosquito Aedes cantans. Medical and Veterinary Entomology 8: 187-193, 1994.

26 Teodoro U, Lonardoni MVC, Silveira TGV, Dias AC, Abbas M, Alberton D, Santos DR. Luz e galinhas como fatores de atração de Nyssomyia whitmani em ambiente rural, Paraná, Brasil. Revista de Saúde Pública 41: 383-388, 2007.

27. Teodoro U, Silveira TGV, Santos DR, Santos ES, Santos AR. Frequiência da fauna de flebotomíneos no domicílio e em abrigos de animais domésticos no peridomicílio, nos municípios de Cianorte e Doutor Camargo, Estado do Paraná, Brasil. Revista de Patologia Tropical 30: 209-223, 2001.

28. Teodoro U, Silveira TGV, Santos DR, Santos ES, Santos AR, Oliveira 0, Kühl JB, Alberton D. Influência da reorganização, da limpeza do peridomicílio e a da desinsetização de edificações na densidade populacional de flebotomíneos no Município de Doutor Camargo, Estado do Paraná, Brasil. Cadernos de Saúde Pública 19: 1801-1813, 2003.

29. Torr SJ, Prior A, Wilson PJ, Schofield S. Is there safety in numbers? The effect of cattle herding on biting risk from tsetse flies. Medical and Veterinary Entomology 21: 301-311, 2007.

30. Vilela EF, Della Lucia TMC. Feromônios de Insetos. Editora Universidade Federal de Viçosa. Viçosa, MG, 1987.

31. World Health Organization, 2007. http://www.int/gb/ebwha/pdf_files/wha60/ A60_10-en.pdf (acessado em 26/01/2008). 